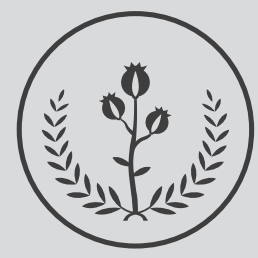

FUCS

\title{
Rept Rep

\section{Prevalencia de dinapenia (disminución de la fuerza), sarcopenia y posibles biomarcadores en rehabilitación cardíaca}

\section{Prevalence of dynapenia (decreased strength), sarcopenia and possible biomarkers in cardiac rehabilitation}

Daniel Alexander Medina MDa Lina Alexandra Laverde $\mathbf{M D}^{\mathbf{a}}$ Luz Karime Alviz MD ${ }^{a}$ Juan Carlos Galvis MD

${ }^{a}$ Medicina de la Actividad Física y del Deporte, Fundación Universitaria De Ciencias de La Salud. Bogotá DC, Colombia.

${ }^{b}$ Director del Programa de Residencia en Medicina de la Actividad Física y del Deporte, Fundación Universitaria de Ciencias de la Salud. Bogotá DC, Colombia.

\section{R E S U M E N}

Introducción: la relación entre dinapenia y sarcopenia da como resultado un aumento adicional en el riesgo de morbimortalidad en la población general, con un incremento progresivo de acuerdo con la edad. A partir de los 30 años debido a la disminución de la masa muscular y de la fuerza, se afecta la capacidad de ejercicio, la calidad de vida y el estado de ánimo. Objetivo: determinar la prevalencia de dinapenia y sarcopenia en los pacientes en rehabilitación cardíaca de un hospital colombiano, con mediciones de la fuerza y de los marcadores bioquímicos. Métodos: estudio de corte transversal en mayores de 40 años en rehabilitación cardíaca con controles al menos una vez por semana. La medición de la fuerza se realizó con dinamómetro de mano, la masa muscular con impedanciómetro bipolar y velocidad de la marcha con la prueba de caminata de 6 metros. Se tomaron mediciones de hormona de crecimiento (GH), testosterona libre, somatomedina IGF-1 y cortisol. Resultados: la disminución de la fuerza muscular fue prevalente en hombres (n=15, 19.4\%; mujeres $\mathrm{n}=5,10 \%)$. La prevalencia de dinapenia fue $15,7 \%$ y sarcopenia $0 \%$. Los resultados obtenidos fueron GH 0.27 (0.08-1.18), testosterona 5.9 (0.3-8.5), IGF-1 126 (95.5-169) cortisol 13.9 (11.2-18.4). Las patologías más frecuentes de los pacientes del programa fueron infarto del miocardio, angioplastia e implantación de stent. Conclusiones: la prevalencia de dinapenia fue $15.7 \%$. No se encontró diferencia alguna de los marcadores bioquímicos en los pacientes con y sin dinapenia.

Palabras clave: composición, dinapenia, músculo, rehabilitación, sarcopenia, rehabilitación.

(C) 2021 Fundación Universitaria de Ciencias de la Salud - FUCS. Este es un artículo Open Access bajo la licencia CC BY-NC-ND (http://creativecommons.org/licenses/by-nc-nd/4.0/).

INFORMACIÓN DEL ARTÍCULO

Historia del artículo:

Fecha recibido: noviembre 20 de 2019 Fecha aceptado: mayo 21 de 2020
Autor para correspondencia. Daniel Alexander Medina damedina2@fucsalud.edu.co
DOI

10.31260/RepertMedCir.01217372.989 
Introduction: the relationship between dynapenia and sarcopenia results in an additional increment in the risk of morbidity and mortality in the general population, with a progressive age-associated increase. From the age of 30, exercise capacity, quality of life and mood are affected due to decreased muscle mass and strength. Objective: to determine the prevalence of dynapenia and sarcopenia in patients in cardiac rehabilitation in a Colombian hospital by measuring their muscle strength and biochemical markers. Methods: a cross-sectional study of patients over 40, in cardiac rehabilitation, attending followup visits at least once a week. Muscle strength was measured with a handheld dynamometer, muscle mass with bipolar impedance and walking speed with the 6-meter walking test. Growth hormone (GH), testosterone, somatomedin IGF-1 levels and control measurements were taken. Results: decrease in muscle strength was prevalent in men $(\mathrm{n}=15,19.4 \%$; women $\mathrm{n}=5,10 \%)$. The prevalence of dynapenia was $15.7 \%$ and of sarcopenia $0 \%$. The results obtained were GH $0.27(0.08-1.18)$, testosterone $5.9(0.3-8.5)$, IGF-1 126 (95.5-169) cortisol 13.9 (11.2-18.4). The most frequent pathological conditions of patients in the program were myocardial infarction, angioplasty and stent implantation. Conclusions: the prevalence of dynapenia was $15.7 \%$. No difference in biochemical markers was found in patients with or without dynapenia.

Key words: composition, dynapenia, muscle, rehabilitation, sarcopenia, rehabilitation.

(C) 2021 Fundación Universitaria de Ciencias de la Salud - FUCS. This is an open access article under the CC BY-NC-ND license (http://creativecommons.org/licenses/by-nc-nd/4.0/).

\section{INTRODUCCIÓN}

La expectativa de vida ha venido en aumento en las últimas décadas debido en parte al mayor acceso a los servicios de salud ${ }^{1}$, esto quiere decir que progresivamente han aumentado las patologías relacionadas con la edad ${ }^{2}$, entre las que se encuentra la dinapenia, que hace referencia a la pérdida de fuerza relacionada con la edad, término proveniente del griego dina qué significa fuerza y penia qué significa pérdida ${ }^{3}$ y la sarcopenia, cuyo término fue acuñado por Rosenberg y derivado del griego sarco "músculo" y penia "pérdida", que es un síndrome caracterizado por la disminución de la fuerza y pérdida progresiva y generalizada de masa muscular esquelética ${ }^{4,5}$ que afecta a más de 50 millones de personas en la actualidad y afectará a más de 200 millones en los próximos 40 años. ${ }^{4}$ Esta prevalencia aumenta en forma progresiva con la edad, así entonces se encuentra entre 5 y $13 \%$ en la séptima década y alcanza de 11 a $50 \%$ en mayores de 80 años. ${ }^{4,6}$ Se inicia a partir de los 30 años con disminución de la masa muscular y la fuerza en cerca de 1 a $2 \%$ cada año. ${ }^{4}$ La inflamación crónica y el aumento en la producción de citoquinas proinflamatorias se ha asociado con la aparición de sarcopenia y además con patologías crónicas como hipertensión, dislipidemia y diabetes mellitus, que son factores de riesgo para las enfermedades cardiovasculares (ECV). ${ }^{7,8}$ Aunque la prevalencia de la sarcopenia relacionada con ECV no ha sido bien definida, se ha estimado un incremento entre 7 y $26.8 \%$ con la edad (<75 años: $16.5 \%, 75-84$ años: $40.3 \%$, $\geq 85$ años: $62.6 \%$ ). ${ }^{9,10}$

El grupo de trabajo europeo de sarcopenia en personas mayores (EWGSOP) en 2010 recomendaba el uso de 3 criterios para establecer el diagnóstico, siendo el principal la disminución de masa muscular, los otros 2 eran fuerza muscular y capacidad física. Así, se requiere del criterio 1 más el 2 o 3 para el diagnóstico de sarcopenia. ${ }^{4,11}$ La presencia de los tres parámetros se considerará sarcopenia severa y cuando es sólo uno presarcopenia. ${ }^{4}$ En la actualización de 2018 (EWGSOP2) se cambiaron los puntos de corte y el orden del diagnóstico, siendo el parámetro principal la fuerza muscular con el que se diagnostica probable sarcopenia. Con el fin de realizar la confirmación del diagnóstico se requiere la disminución de la masa muscular y para valorar la gravedad debe haber reducción del rendimiento físico. ${ }^{5}$

Para lograr el diagnóstico de sarcopenia existen múltiples métodos disponibles para la medición de la masa muscular esquelética, como antropometría, metabolitos urinarios, ultrasonido, análisis de bioimpedancia e imágenes como tomografía computarizada (TC), absorciometría de rayos $\mathrm{X}$ de energía dual (DXA) y resonancia magnética (RM). ${ }^{12}$ El más utilizado en investigación y diagnóstico en entornos de alto recurso es $\mathrm{DXA}^{13}$, pero su costo es elevado y no siempre está disponible. Una alternativa es el análisis de bioimpedancia (BIA), que es económico, seguro, portátil y útil para el monitoreo a largo plazo. ${ }^{12}$ Los sistemas BIA cuantifican los compartimentos corporales basándose en las diferencias de la resistencia al flujo de la corriente cuando se aplica una tensión eléctrica. En la actualidad, algunos estudios informan "buenas correlaciones entre la masa de músculo esquelético apendicular derivada de BIA y las estimaciones de tejido blando DXA magro". ${ }^{12}$ Por lo tanto, 
la BIA es una opción para la medición de la masa muscular esquelética en países de bajos y medianos ingresos, no solo porque está disponible y asequible y sus mediciones tienen una buena correlación con las estimaciones DXA.

El grupo EWGSOP2 recomienda marcadores inflamatorios de estrés oxidativo, nutricionales y hormonales como biomarcadores de sarcopenia. ${ }^{4}$ Después de los 50 años en el hombre y 45 años en mujeres la presencia de resistencia anabólica se traduce en disminución de la capacidad de síntesis muscular y por ende la aparición de sarcopenia ${ }^{14}$, dado que la edad se relaciona con disminución de niveles de testosterona que tiene papel en la estimulación de los mioblastos, inhibición de la miostatina y aumento de las células satélite. Por ello bajar los niveles de testosterona disminuye el IGF-1 (potente activador de la vía AKT), disminuyen los niveles de hormona de crecimiento $(\mathrm{GH})^{15}$ y se presenta alteración de los marcadores inflamatorios como IL-6.6 Esa disminución de hormonas anabólicas va acompañada de menor masa muscular y fuerza. ${ }^{16,17}$ Los niveles de testosterona disminuyen en forma gradual en cerca de $1 \%$ por año y la testosterona biodisponible en alrededor de $2 \%$ por año en los hombres después de alcanzar la edad 30 años de vida. ${ }^{18,19}$ El descenso de testosterona, dehidroepiandrosterona, GH y IGF-1 están implicados en el proceso de sarcopenia.

La reducción de la fuerza y de la masa muscular aumenta la morbilidad y la mortalidad, reduce la capacidad de ejercicio, afecta la calidad de vida y perturba el estado de ánimo ${ }^{20}$, observando que la población con ECV está fuertemente asociada. En la actualidad se desconoce la prevalencia de dinapenia en pacientes colombianos sometidos a rehabilitación cardíaca. Por lo tanto, el objetivo de este estudio fue determinar la prevalencia de la disminución de la fuerza (dinapenia o probable sarcopenia según el grupo europeo) y sarcopenia en un grupo de pacientes sometidos a rehabilitación cardíaca en un hospital colombiano, de acuerdo con las mediciones de fuerza muscular con dinamometría de brazo, la masa muscular esquelética con un impedanciómetro bipolar y el punto de corte determinado después del análisis de datos y el hallado para nuestra población según nuestros resultados.

\section{MÉTODOS}

\section{Participantes}

Estudio de corte transversal que incluyó pacientes que asistían al programa de rehabilitación y prevención cardiovascular del servicio de actividad física y medicina del deporte del Hospital Infantil Universitario de San José durante 11 meses. Todos fueron mayores de 40 años que se sometieron a rehabilitación cardíaca o sesiones de prevención cardiovascular al menos una vez a la semana y que eran capaces de proporcionar un consentimiento informado por escrito. Se excluyeron aquellos con trastornos neurológicos, enfermedades neoplásicas, amputaciones o limitaciones funcionales en sus extremidades superiores o inferiores, con presencia de tubos de alimentación, colostomías, marcapasos implantables, dispositivos de desfibrilación cardíaca o dependencia física de un miembro de la familia.

La investigación fue aprobada por el comité de ética e investigación con seres humanos del Hospital de San José de Bogotá y se acogió a las normas de buenas prácticas clínicas en investigación, garantizando la confidencialidad de la información en la recolección y análisis de los datos, así como a la resolución 8430 de 1993 de Colombia, según la cual el estudio fue clasificado como de riesgo mínimo.

\section{Fuente de los datos}

El peso corporal y la altura se midieron con una precisión de $0,1 \mathrm{~kg}$ y $0,1 \mathrm{~cm}$ utilizando equipos y procedimientos estandarizados. La BIA se obtuvo utilizando un impedanciómetro bipolar (Tanita BC554 Ironman Glass InnerScan Body Composition Monitor Elite Series).

\section{Diagnóstico de dinapenia}

De acuerdo con el primer criterio de EWGSOP2 la medición precisa de la fuerza de agarre requiere el uso de un dinamómetro calibrado de mano. Se realizó la medición de las dos manos y se tomó el resultado de la derecha para el análisis. ${ }^{5}$

\section{Diagnóstico de sarcopenia}

Los criterios utilizados fueron los propuestos por EWSGOP2, teniendo en cuenta la medición de fuerza, masa muscular y el rendimiento en la marcha. ${ }^{5}$ Los valores se compararon con los datos de referencia y se estratificaron por género y si tenían diagnóstico de probable sarcopenia, sarcopenia y sarcopenia grave, definida la primera como disminución de la fuerza muscular (dinamometría < $27 \mathrm{k}$ en hombres y $<16 \mathrm{k}$ en mujeres), la sarcopenia se estableció como la baja masa de músculo esquelético $(<7$ $\mathrm{k} / \mathrm{m} 2$ en hombres y $<6 \mathrm{k} / \mathrm{m} 2$ en mujeres) asociada con la disminución de la fuerza, y la sarcopenia grave se definió como la disminución de la fuerza asociada con pérdida de masa muscular esquelética y rendimiento funcional deficiente (velocidad de caminata $\leq 0.8 \mathrm{~m} / \mathrm{s}$ ). ${ }^{5}$

\section{Medición de la masa muscular}

La medición de la masa muscular se realizó utilizando un impedanciómetro bipolar (Tanita BC554 Ironman Glass InnerScan Body Composition Monitor Elite Series), se les explico a los pacientes las condiciones recomendadas para la medición: ayuno durante 4 horas, vejiga vacía y no haber realizado ejercicio en las 8 horas anteriores, y el cálculo de la masa muscular se hizo tomando el valor absoluto en $\mathrm{k}$ 
(masa muscular absoluta) para realizar el cálculo del IMME (índice de masa muscular esquelética) tomando este valor y dividiéndolo en la talla al cuadrado $(\mathrm{k} / \mathrm{m} 2)$. Los valores límite de IMME para hombres y mujeres fueron $<7 \mathrm{y}<6 \mathrm{k} /$ $\mathrm{m} 2$, respectivamente. ${ }^{5}$

\section{Medición de la fuerza}

La fortaleza muscular se evaluó con la fuerza de la empuñadura (HGS), utilizando un dinamómetro de mano calibrado (CAMRY®EH101). Se realizó medición en las dos manos y se usó el resultado de la derecha para el análisis, se tomaron como referencia los criterios de EWGSOP2, los puntos de corte de HGS eran $<27 \mathrm{k} \mathrm{y}<16 \mathrm{k}$ para hombres y mujeres, respectivamente. ${ }^{5}$

\section{Medición de la marcha}

El rendimiento físico se valoró con la prueba de caminata de 6 metros con inicio de carrera detenida, la cual se calcula dividiendo la distancia recorrida en metros sobre el tiempo utilizado, los pacientes con una velocidad de la marcha $\leq 0,8 \mathrm{~m} / \mathrm{s}$ se consideraron con bajo rendimiento físico. ${ }^{4,5}$

\section{Pruebas de laboratorio}

Se recolectaron las muestras en un laboratorio particular, a las 8 de la mañana bajo ayuno, con valores de referencia para la hormona de crecimiento (GH) 0,4-10 ng/mL, testosterona 10.8 - $24.6 \mathrm{pg} / \mathrm{mL}$ en hombres y $0.14-1.8 \mathrm{pg} /$ $\mathrm{mL}$ mujeres, para IGF1 17-206 ng/mL en hombres y 17-241 $\mathrm{ng} / \mathrm{mL}$ mujeres y cortisol 8-19 ug/dL.

\section{Análisis estadístico}

Los datos obtenidos se tabularon utilizando excel 2007 (Microsoft, 171 Redmond, WA, EE. UU.) Se realizaron análisis estadísticos con stata versión 13.0 (StataCorp. 2007, College Station, TX: StataCorp LP). Las variables cuantitativas se describieron con medidas de tendencia central (promedio y mediana) y de dispersión, de acuerdo con su distribución de normalidad (prueba de ShapiroWilk), mientras que las variables cualitativas se expresaron como frecuencias relativas y valores absolutos.

\section{RESULTADOS}

Se incluyeron en el estudio 127 pacientes de los cuales 77 $(60.6 \%)$ eran varones. La edad media fue $64( \pm 10,7)$ años. Los principales diagnósticos de enfermedad cardiovascular de la población fueron: infarto de miocardio $(n=61,47 \%)$, hipertensión $(\mathrm{n}=9,7 \%)$ y enfermedad coronaria $(\mathrm{n}=9$, $7 \%$ ). Los diagnósticos secundarios asociados fueron angioplastia y colocación de endoprótesis ( $\mathrm{n}=30,23 \%)$, hipertensión ( $\mathrm{n}=26,20 \%)$ y revascularización miocárdica $(\mathrm{n}=10,7,8 \%)$. Otras características de los pacientes se muestran en la (tabla 1).
Tabla 1. Características sociodemográficas y clínicas

\begin{tabular}{|c|c|c|c|}
\hline Variable & $\begin{array}{c}\text { Sin sarcopenia } \\
n=107\end{array}$ & $\begin{array}{c}\text { Dinapenia } \\
n=20\end{array}$ & Total n=127 \\
\hline $\begin{array}{c}\text { Edad, años } \\
\text { Promedio, DE } \\
\text { Rango (min-max) }\end{array}$ & $\begin{array}{c}63.7( \pm 10.5) \\
40-84\end{array}$ & $\begin{array}{c}64.7( \pm 11.5) \\
42-80\end{array}$ & $\begin{array}{c}64( \pm 10.7) \\
40-84\end{array}$ \\
\hline $\begin{array}{l}\text { Sexo }(\mathrm{n}, \%) \\
\text { Hombres }\end{array}$ & $63(58.8)$ & $14(70)$ & $77(60.6)$ \\
\hline $\begin{array}{c}\text { Estrato } \\
\qquad \begin{array}{c}1 \\
2 \\
3 \\
4 \\
5\end{array}\end{array}$ & $\begin{array}{c}5(4.6) \\
23(21-5) \\
65(60.7) \\
11(10.2) \\
3(2.8)\end{array}$ & $\begin{array}{c}1(5) \\
7(35) \\
8(40) \\
2(10) \\
2(10)\end{array}$ & $\begin{array}{c}6(4.7) \\
30(23.6) \\
57.4(73) \\
13(10.2) \\
5(3.9)\end{array}$ \\
\hline $\begin{array}{c}\text { Talla, } \mathrm{m} \\
\text { Promedio, DE } \\
\text { Rango (min-max) }\end{array}$ & $\begin{array}{c}1.62( \pm 0,07) \\
1.47-1.78\end{array}$ & $\begin{array}{c}1.61( \pm 0.05) \\
1.5-1.7\end{array}$ & $\begin{array}{c}1.62( \pm 0,07) \\
1.47-1.78\end{array}$ \\
\hline $\begin{array}{c}\text { Índice de masa } \\
\text { corporal (IMC), k/m2 } \\
\text { Mediana, RIQ } \\
\text { Rango (min-max) }\end{array}$ & $\begin{array}{c}25.6(23.5-29) \\
20.4-42\end{array}$ & $\begin{array}{c}23.7(22.9-27.1) \\
20-29.8\end{array}$ & $\begin{array}{c}25.2(23.2-28.6) \\
20-42\end{array}$ \\
\hline $\begin{array}{l}\text { Porcentaje masa grasa } \\
\text { Promedio, DE }\end{array}$ & $29.3( \pm 8.2)$ & $25.9( \pm 7.8)$ & $28,7( \pm 8.2)$ \\
\hline $\begin{array}{c}\text { Diagnósticos ECV, n(\%) } \\
\text { IAM } \\
\text { HTA } \\
\text { Dislipidemia }\end{array}$ & $\begin{array}{c}56(55.4) \\
14(13.8) \\
2(2)\end{array}$ & $\begin{array}{c}8(42.1) \\
2(10.5) \\
0\end{array}$ & $\begin{array}{c}64(53.3) \\
16(13.3) \\
2(1.6)\end{array}$ \\
\hline Enfermedad coronaria & $13(12,8)$ & $4(21)$ & $17(14.1)$ \\
\hline Valvulopatía & $5(4.9)$ & $2(10.5)$ & $7(5.8)$ \\
\hline $\begin{array}{l}\text { Síncope vaso vagal } \\
\text { Síncope } \\
\text { neurocardiogénico }\end{array}$ & $\begin{array}{l}5(4.9) \\
3(2.9)\end{array}$ & $\begin{array}{l}0 \\
0\end{array}$ & $\begin{array}{l}5(4.1) \\
3(2.5)\end{array}$ \\
\hline $\begin{array}{l}\text { Angina de pecho } \\
\text { Falla cardiaca }\end{array}$ & $\begin{array}{c}3(2.9) \\
0\end{array}$ & $\begin{array}{c}1(5.2) \\
2(10.5)\end{array}$ & $\begin{array}{l}4(3.3) \\
2(1.6)\end{array}$ \\
\hline $\begin{array}{l}\text { Peso muscular, k } \\
\text { Mediana, RIQ }\end{array}$ & $31.4(23.6-39.1)$ & $27.9(22.5-33.1)$ & $30.9(23.6-38.6)$ \\
\hline
\end{tabular}

Definición EWGSOP2 de 2018 (conocido como probable sarcopenia).

DE: Desviación Estándar; RIQ: rango inter cuartilico; ECV: enfermedad cardiovascular; IAM: infarto agudo del miocardio; HTA: hipertensión arterial.

Fuente: Ios autores.

Entre las características para el diagnóstico clínico de sarcopenia se encontró que 5 mujeres (10\%) tenían dinamometría de agarre <16 k y 15 (19,4\%) hombres por debajo de $27 \mathrm{k}$, la mediana del IMME de las mujeres fue de 9.4 k / m2 (RIQ 8.4-11.6) y para los hombres 12,8 k / m2 (RIQ 11.1-15) (tabla 2). El rendimiento físico evaluado mediante una prueba de marcha de 6 metros, mostró que $12(24 \%)$ mujeres y $13(16,8 \%)$ hombres tenían una velocidad de marcha $\leq 0,8 \mathrm{~m} / \mathrm{s}$ (tabla 2). Los resultados de GH fueron 0.27 (0.08-1.18), testosterona 5.9 (0.3-8.5), IGF-1 126 (95.5-169) y cortisol 13.9 (11.2-18.4), que se muestran en la (tabla 3).

La prevalencia de dinapenia en los pacientes fue $15,7 \%$, cinco mujeres (10\%) 15 hombres (19,4\%); ningún participante cursó con sarcopenia o sarcopenia severa ya que no se hallaron valores disminuidos de IMME. 
Tabla 2. Criterios para diagnóstico clínico en pacientes que asisten a programa de rehabilitación cardiaca

\begin{tabular}{|c|c|c|c|c|}
\hline Variable & Total $n=127$ & $\underset{\substack{\text { Sin } \\
\mathrm{n}=10}}{\operatorname{sinapenia}}$ & $\underset{n=20}{\text { Dinapenia* }}$ & Valor de $\mathrm{p}^{\text {** }}$ \\
\hline $\begin{array}{l}\text { Índice de masa muscular esquelética(IMME), k/m2 } \\
\text { Mediana, RIQ }\end{array}$ & $11.8(9.5-14.1)$ & $12(9.5-14)$ & $10.9(9.3-12.6)$ & 0.22 \\
\hline $\begin{array}{c}\text { IMME mujeres, k/m2 } \\
\text { Mediana, RIQ }\end{array}$ & $9.4(8.4-11.6)$ & $9.7(8.7-11.8)$ & $8.8(8.3-9.4)$ & 0.29 \\
\hline $\begin{array}{l}\text { IMME hombres, } \mathrm{k} / \mathrm{m} 2 \\
\text { Mediana, RIQ }\end{array}$ & $12.8(11.1-15)$ & $13.2(11.3-15.2)$ & $11.7(10.3-13.2)$ & 0.11 \\
\hline $\begin{array}{l}\text { Dinamometría de mano derecho mujeres, } \mathrm{k} \\
\text { Mediana,RIQ }\end{array}$ & $23(19-30)$ & $24(20-30)$ & $13(11-26)$ & 0.03 \\
\hline $\begin{array}{l}\text { Dinamometría de mano derecho hombres, } \mathrm{k} \\
\text { Mediana, RIQ }\end{array}$ & $36(30-40)$ & $38(31-42)$ & $22(20-40)$ & 0.00 \\
\hline $\begin{array}{c}\text { Velocidad de la marcha en test de caminata de } 6 \text { metros } \\
\text { mujeres, } \mathrm{m} / \mathrm{s} \\
\text { Mediana, } \mathrm{RIQ}\end{array}$ & $1.05(0.8-1.1)$ & 0.99(0.78-1.1) & $1.2(0.95-1.4)$ & 0.21 \\
\hline $\begin{array}{c}\text { Velocidad de la marcha en test de caminata de } 6 \text { metros } \\
\text { en hombres, } \mathrm{m} / \mathrm{s} \\
\text { Mediana,RIQ }\end{array}$ & $1.1(0.94-1.3)$ & $1.1(0.94-1.3)$ & $0.98(0.94-1.1)$ & 0.07 \\
\hline
\end{tabular}

${ }^{*}$ Corresponde a pacientes diagnosticados con probable $(n=20) ;{ }^{* *}$ Prueba Mann-Whitney. Fuente: los autores.

Tabla 3. Niveles hormonales de pacientes que asisten a programa de rehabilitación cardiaca

\begin{tabular}{|c|c|c|c|c|}
\hline Variable & Total $n=127$ & $\underset{n=10}{\text { Sin dinapenia }}$ & $\underset{n=20}{\text { Dinapenia* }}$ & Valor de $p^{* *}$ \\
\hline $\begin{array}{c}\text { Testosterona Libre }(\mathrm{pg} / \mathrm{mL}) \\
\text { Mediana, RIQ } \\
\text { Mujeres } \\
\text { Hombres }\end{array}$ & $5.9(0.3-8.5)$ & $\begin{array}{c}5.9(0.3-8.5) \\
0.25(0.14-0.5) \\
7.7(6.3-9.8)\end{array}$ & $\begin{array}{l}6.9(0.7-8.5) \\
0.2(0.1-0.5) \\
7.9(6.2-8.9)\end{array}$ & $\begin{array}{l}0.45 \\
0.89 \\
0.91\end{array}$ \\
\hline $\begin{array}{l}\text { Hormona de Crecimiento GH (ng/dl) } \\
\text { Mediana, RIQ }\end{array}$ & $0.27(0.08-1.18)$ & $0.29(0.08-1.3)$ & $0.7(0.1-4.5)$ & 0.19 \\
\hline $\begin{array}{c}\text { Somatomedina C (IGF-1) }(\mathrm{ng} / \mathrm{mL}) \\
\text { Mediana, RIQ } \\
\text { Mujeres } \\
\text { Hombres }\end{array}$ & $126(95.5-169)$ & $\begin{array}{c}131(95.6-170) \\
125.5(96-178.5) \\
136(95.6-170)\end{array}$ & $\begin{array}{c}113.5(85.5-154) \\
102.8(0.1-137) \\
117(97.8-157)\end{array}$ & $\begin{array}{l}0.15 \\
0.31 \\
0.33\end{array}$ \\
\hline $\begin{array}{c}\text { Cortisol (ug/dL) } \\
\text { Mediana, RIQ }\end{array}$ & $13.9(11.2-18.4)$ & $13.9(11.1-18.3)$ & 14.1(12.2-18.9) & 0.72 \\
\hline
\end{tabular}

${ }^{*}$ RIQ: rango inter cuartilico, IGF-1: *prueba Mann-Whitney. Fuente: Ios autores.

\section{DIS C USIÓN}

Hay consenso en cuatro definiciones principales de sarcopenia que incluyen medición del tamaño muscular y otra función muscular ${ }^{12}$ : el grupo de trabajo europeo sobre sarcopenia en mayores (EWGSOP), el internacional sobre sarcopenia (IWGS), la fundación del Instituto Nacional de Salud (FNIH) y otros grupos de interés especial como la Sociedad Europea de Nutrición Clínica y Metabolismo (ESPEN). ${ }^{4,5,13}$ Dependiendo de la definición utilizada, la prevalencia de la sarcopenia varía. Hasta la fecha no hay informes sobre la frecuencia en esta enfermedad en pacientes colombianos que se someten a un programa de rehabilitación cardiaca ni de sus mediciones bioquímicas.

Es bien sabido que la sarcopenia juega un papel crítico en la patogenia de la fragilidad y las limitaciones funcionales en los adultos mayores y en los pacientes con ECV. ${ }^{21}$ También se asocia con un mayor riesgo de resultados adversos como discapacidad física, mala calidad de vida, dependencia, morbilidad y mortalidad. ${ }^{4,13,22,23}$ Por lo tanto, el diagnóstico precoz permite el inicio inmediato del tratamiento y tiene como objetivo disminuir los resultados adversos asociados con la pérdida de la fuerza, la masa muscular esquelética y el rendimiento físico.
La sarcopenia se diagnostica cuando hay disminución de la fuerza muscular asociada con una baja masa muscular esquelética. En el presente estudio la fuerza se midió mediante dinamometría de agarre ( $<27 \mathrm{k}$ para hombres, $<16 \mathrm{k}$ mujeres), la masa muscular esquelética se evaluó mediante BIA $(<7 \mathrm{k} /$ $\mathrm{m}^{2}$ para hombres; $<6 \mathrm{k} / \mathrm{m}^{2}$ mujeres) y el rendimiento físico se valoró por la velocidad de la marcha $(<0,8 \mathrm{~m} / \mathrm{s}$, para ambos sexos). ${ }^{5}$ En nuestros resultados, 20 de los 127 pacientes tenían disminución de la fuerza muscular (5 mujeres y 15 hombres), sin encontrar baja masa muscular en los participantes; 12 mujeres y 13 hombres tuvieron una velocidad de la marcha $\leq 0,8 \mathrm{~m} / \mathrm{s}$. Dado que con la modificación del diagnóstico propuesto por el EWGSOP2 en 2018, la disminución de la fuerza muscular es imprescindible para la sospecha $\mathrm{y}$ el diagnóstico de sarcopenia, este estudio mostró que la prevalencia del síndrome clínico de sarcopenia entre todos los pacientes fue de $0 \%$, por consiguiente no hay casos de sarcopenia grave, pero es probable el diagnóstico de sarcopenia o dinapenia en $15.7 \%$.

La disminución de la fuerza muscular fue prevalente en hombres $(19,4 \%$ vs $10 \%)$ sin evidencia de baja masa del músculo esquelético. Ante este hallazgo surge la hipótesis de que los parámetros con los cuales nos basamos fueron 
los realizados por la EWGSOP2 con europeos y puede ser posible que nuestra población tenga un promedio mayor de masa muscular pues aún no contamos con valores de referencia para Colombia, ni para la población con ECV, por lo cual y como lo sugirió la EWGSOP2 debemos validar esos valores con nuestras medidas. Ante esto tomamos 2 DE por debajo de la media para calcular cifras para nuestra población siendo para mujeres un promedio de 10.34, realizando el cálculo sobre $2 \mathrm{DE}$ por debajo dando un punto de corte de 5.04 y para los hombres un promedio de 13,33, con 2 DE por debajo de 7,04, siendo este último dato muy cercano a las guías europeas, y para las mujeres una cifra muy inferior, aún por debajo de las guías. Con ello no obtenemos ningún valor que demuestre masa muscular disminuida. Los resultados son tan diversos pues las mediciones que realizamos fueron con impedanciómetro bipolar y los estudios internacionales utilizan los tetrapolares, por lo que no son comparables los resultados y se puede estar sobreestimando la IMME. Además, se necesitan más estudios con la gente mayor en Colombia.

En los resultados de laboratorio (tabla 3) observamos niveles disminuidos de testosterona, IGF-1 y hormona de crecimiento que se relacionan con el diagnóstico probable de sarcopenia y concuerdan con los resultados obtenidos por el estudio realizado por Ferrini y col. ${ }^{24}$ donde con la edad los niveles de hormonas sexuales como estradiol y testosterona disminuyeron, pero ninguno con valor $\mathrm{p}$ significativo. Los niveles de hormona de crecimiento se encontraron bajos pero con medianas mayores en el grupo de probable sarcopenia, lo que es opuesto a la teoría que con la edad disminuye la GH, como fenómeno secundario a la disminución del ambiente anabólico. ${ }^{25}$ En cuanto a los niveles de IGFl se evidenció disminución en los niveles comparando el brazo de probable sarcopenia con los que no tienen diagnóstico de dinapenia aunque con un valor p 0.15, y los niveles de cortisol se hallaron aumentados en el grupo de sarcopenia con una $\mathrm{p}$ 0.72 , lo cual se podría relacionar con la teoría de la sarcopenia como un estado proinflamatorio. ${ }^{25}$

Se sabe que la función del eje de la hormona del crecimiento (GH) / IGF-1 y los niveles de testosterona disminuyen con el envejecimiento ${ }^{26}$ y que el IGF-1 activa la vía AKT-MTOR para la hipertrofia muscular. ${ }^{27}$ Dado que hay niveles bajos de estas hormonas en las personas mayores y en especial en los hombres, suponemos que es más difícil estimular la hipertrofia muscular y preservar la masa muscular en esta población, lo que podría explicar la mayor prevalencia de sarcopenia en los hombres mayores. Sin embargo, se necesitan más estudios para establecer una relación más directa entre los niveles de GH, IGF-1 y testosterona y la baja masa muscular en pacientes en rehabilitación cardíaca.

Existen estudios en Colombia que informan sobre la prevalencia de sarcopenia en hogares de $\operatorname{ancianos}^{28} \mathrm{y}$ en pacientes de 60 años o más en Bogotá $^{23}$, de 38.9\% y 6.96\%, respectivamente. En otros países la prevalencia oscila entre $5 \%$ en Estados Unidos, 6\% en Canadá, $7.8 \%$ en Taiwán, $10.2 \%$ en Italia, $12.5 \%$ en Bélgica y $24.2 \%$ en Japón. ${ }^{26}$ La mayoría de estos estudios se realizaron en pacientes geriátricos. Nuestros datos revelan que la prevalencia de sarcopenia con un impedanciómetro bipolar en pacientes en rehabilitación cardíaca en Bogotá es de 0\%. Sin embargo, es importante recordar que existen múltiples definiciones y criterios para diagnosticar la sarcopenia, y probablemente estos resultados no sean comparables.

Los pacientes que asisten a los programas de rehabilitación cardíaca por lo regular son adultos mayores, no realizan actividad física regular y tienen diferentes comorbilidades que afectan la masa muscular. En este estudio, las patologías más prevalentes asociadas con pacientes en rehabilitación cardíaca fueron infarto de miocardio, angioplastia y colocación de stent, revascularización miocárdica, enfermedad coronaria, hipertensión y dislipidemia. La mayoría de estas patologías están asociadas con comportamientos poco saludables del estilo de vida, como la mala alimentación, la inactividad física, el consumo de alcohol, el tabaquismo y la participación social deficiente. ${ }^{29,30}$ Además, todos aumentan el riesgo de morbilidad y mortalidad a corto, medio y largo plazo.

Es claro que el rendimiento físico en todos es aceptable, lo que significa que están con capacidad física aceptable para su edad y condición de salud. Como los programas de rehabilitación cardiaca están dirigidos a mejorar el rendimiento físico, todos los pacientes mejoran la técnica de marcha adquiriendo mejoras en la velocidad y la resistencia, por lo tanto no es fácil encontrar un bajo rendimiento físico en quienes están en los programas de rehabilitación cardiaca. $^{31}$

Los que ingresan a programas de rehabilitación cardíaca tienen riesgos cardiovasculares variables asociados con su diagnóstico principal y/o comorbilidades. Ante la prevalencia de dinapenia de $15.7 \%$ sin sarcopenia o sarcopenia grave, recomendamos que todos los que entren en programas de rehabilitación cardíaca deben ser evaluados en busca de parámetros para el diagnóstico del síndrome clínico de la sarcopenia, con el fin de prevenir las posibles complicaciones asociadas con la disminución de la fuerza y la masa muscular. Al establecer el diagnóstico y tratamiento oportunos se podrían modificar los hábitos y el estilo de vida, en especial la actividad física y la nutrición para brindar una mejor esperanza y calidad de vida.

Reconocemos que probablemente se necesite una muestra más grande para conocer la prevalencia de sarcopenia en los pacientes en rehabilitación cardíaca. La fortaleza del estudio fue evaluar a un grupo que se encontraba en un 
programa de rehabilitación cardiaca, a pesar de no contar en Colombia con información estadística de prevalencia en nuestra población. Entre las debilidades está el método de medición de la masa muscular ya que solo contamos con impedanciómetro bipolar y los informes internacionales se realizan con uno tetrapolar, lo cual puede sobreestimar los valores de masa muscular y ser la causa de no encontrar ningún caso de sarcopenia en nuestra población a estudio.

\section{CONCLUSIÓN}

La prevalencia de dinapenia en un centro de rehabilitación cardíaca en Bogotá, Colombia, fue de $15.7 \%$, siendo más frecuente en hombres que en mujeres $(19.4 \%$ vs $10 \%)$, además no se hallaron casos de sarcopenia ni sarcopenia severa, ya que ningún paciente presentó disminución de masa muscular de acuerdo con los parámetros de la EWGSOP2. Por ello, planteamos modificar nuestro punto de corte teniendo en cuenta $2 \mathrm{DE}$ por debajo del promedio obtenido en el estudio, con resultado 5,04 para mujeres y 7,04 para hombres, niveles bajos con los que no obtuvimos resultado alguno que indicara baja masa muscular.

Dado que BIA ha demostrado una buena correlación con DXA y es más económica, la medición de la masa muscular esquelética con un impedanciómetro bipolar ofrece una alternativa para entornos de bajos recursos en Colombia y otros países de ingresos bajos y medianos, donde DXA no está fácilmente disponible ni asequible, pero teniendo en cuenta que no es el instrumento utilizado en los estudios internacionales, puede que los datos obtenidos no sean comparables. No se encontraron diferencias significativas para el diagnóstico bioquímico de la sarcopenia, lo cual está de acuerdo con la literatura donde los resultados no son concluyentes. Hacen falta estudios más grandes en nuestra población para estandarizar los criterios de IMME. Recomendamos que el síndrome clínico de sarcopenia se debe evaluar y tratar en todos los pacientes que asisten a los programas de rehabilitación cardíaca y realizar estudios con una mayor población y/o multicéntrico para poder calcular valores de masa muscular esquelética de acuerdo con las guías.

\section{DECLARACIÓN DE CONFLICTO DE INTERÉS}

Los autores declaran no tener ningún conflicto de intereses.

DECLARACIÓN DE FINANCIACIÓN DEL PROYECTO

No se recibió financiación.

\section{REFEREN CIAS}

1. Ricci WM, Brandt A, McAndrew C, Gardner MJ. Factors affecting delay to surgery and length of stay for patients with hip fracture. J Orthop Trauma. 2015;29(3):e109-14. doi: https://doi.org/10.1097/ BОT.0000000000000221

2. Borghans I, Kleefstra SM, Kool RB, Westert GP. Is the length of stay in hospital correlated with patient satisfaction?. Int J Qual Heal Care. 2012;24(5):443-51. doi: https://doi.org/10.1093/intqhc/ mzs037

3. Clark BC, Manini TM. Sarcopenia =/= dynapenia. The journals of gerontology Series A, Biological sciences and medical sciences. 2008;63(8):829-34. doi: https://doi.org/10.1093/gerona/63.8.829

4. Cruz-Jentoft AJ, Baeyens JP, Bauer JM, Boirie Y, Cederholm T, Landi F, et al. Sarcopenia: European consensus on definition and diagnosis: Report of the European Working Group on Sarcopenia in Older People. Age Ageing. 2010;39(4):412-23. doi: https://doi. org/10.1093/ageing/afq034

5. Cruz-Jentoft AJ, Bahat G, Bauer J, Boirie Y, Bruyere O, Cederholm $\mathrm{T}$, et al. Sarcopenia: revised European consensus on definition and diagnosis. Age Ageing. 2019;48(1):16-31. doi: https://doi. org/10.1093/ageing/afyl69

6. Ali S, Garcia JM. Sarcopenia, cachexia and aging: diagnosis, mechanisms and therapeutic options - a mini-review. Gerontology. 2014;60(4):294-305. doi: https://doi.org/10.1159/000356760

7. Harada H, Kai H, Shibata R, Niiyama H, Nishiyama Y, Murohara $\mathrm{T}$, et al. New diagnostic index for sarcopenia in patients with cardiovascular diseases. PloS one. 2017;12(5):e0178123. doi: https://doi.org/10.1371/journal.pone.0178123.t001

8. Han P, Yu H, Ma Y, Kang L, Fu L, Jia L, et al. The increased risk of sarcopenia in patients with cardiovascular risk factors in SuburbDwelling older Chinese using the AWGS definition. Sci Rep. 2017;7(1):9592. doi: http://dx.doi.org/10.1038/s41598-017-084888

9. Tanaka S, Kamiya K, Hamazaki N, Matsuzawa R, Nozaki K, Maekawa E, et al. Utility of SARC-F for Assessing Physical Function in Elderly Patients With Cardiovascular Disease. J Am Med Dir Assoc. 2017;18(2):176-81. doi: https://doi.org/10.1016/j. jamda.2016.10.019

10. Chin SO, Rhee SY, Chon S, Hwang YC, Jeong IK, Oh S, et al. Sarcopenia is independently associated with cardiovascular disease in older Korean adults: the Korea National Health and Nutrition Examination Survey (KNHANES) from 2009. PloS one. 2013;8(3):e60119. doi: https://doi.org/10.1371/journal. pone.0060119

11. Bonnefoy M, Berrut G, Lesourd B, Ferry M, Gilbert T, Guerin O, et al. Frailty and nutrition: searching for evidence. J Nutr Heal Aging. 2015;19(3):250-7. doi: https://doi.org/10.1007/s12603-0140568-3

12. Heymsfield SB, Gonzalez MC, Lu J, Jia G, Zheng J. Skeletal muscle mass and quality: evolution of modern measurement concepts in the context of sarcopenia. Proc Nutr Soc. 2015;74(4):355-66. doi: https://doi.org/10.1017/S0029665115000129 
13. Edwards MH, Buehring B. Novel Approaches to the Diagnosis of Sarcopenia. Journal of clinical densitometry : the official journal of the International Society for Clinical Densitometry. 2015;18(4):4727. Doi: http://dx.doi.org/10.1016/j.jocd.2015.04.010

14. Keller K. Sarcopenia. Wien Med Wochenschr. 2019;169(7-8):15772. doi: https://doi.org/10.1007/s10354-018-0618-2

15. Shin MJ, Jeon YK, Kim IJ. Testosterone and Sarcopenia. The world journal of men's health. 2018;36(3):192-8. doi: https://doi. org/10.5534/wjmh.180001

16. Baumgartner RN, Waters DL, Gallagher D, Morley JE, Garry PJ. Predictors of skeletal muscle mass in elderly men and women. Mech Ageing Dev. 1999;107(2):123-36. doi: https://doi. org/10.1016/s0047-6374(98)00130-4

17. Morley JE, Baumgartner RN, Roubenoff R, Mayer J, Nair KS. Sarcopenia. The Journal of laboratory and clinical medicine. 2001;137(4):231-43. doi: https://doi.org/10.1067/mlc.2001.113504

18. Burton LA, Sumukadas D. Optimal management of sarcopenia. Clin Interv Aging. 2010;5:217-28. doi: https://doi.org/10.2147/ cia.s11473

19. Lapauw B, Goemaere S, Zmierczak H, Van Pottelbergh I, Mahmoud A, Taes $\mathrm{Y}$, et al. The decline of serum testosterone levels in community-dwelling men over 70 years of age: descriptive data and predictors of longitudinal changes. Eur J Endocrinol. 2008;159(4):459-68. doi: https://doi.org/10.1530/EJE-07-0873

20. Schopfer DW, Forman DE. Cardiac Rehabilitation in Older Adults. The Can J Cardiol. 2016;32(9):1088-96. doi: http://dx.doi. org/10.1016/j.cjca.2016.03.003

21. Harada H, Kai H, Niiyama H, Nishiyama Y, Katoh A, Yoshida N, et al. Effectiveness of Cardiac Rehabilitation for Prevention and Treatment of Sarcopenia in Patients with Cardiovascular Disease - A Retrospective Cross-Sectional Analysis. J Nutr Heal Aging. 2017;21(4):449-56. doi: http://dx.doi.org/10.1007/s12603-0160743-9

22. Falcon LJ, Harris-Love MO. Sarcopenia and the New ICD-10CM Code: Screening, Staging, and Diagnosis Considerations. Fed Pract. 2017;34(7):24-32.

23. Patino-Hernandez D, David-Pardo DG, Borda MG, Perez-Zepeda MU, Cano-Gutierrez C. Association of Fatigue With Sarcopenia and its Elements: A Secondary Analysis of SABE-Bogota. Gerontol
Geriatr Med. 2017;3:2333721417703734. doi: http://dx.doi. org/10.1177/2333721417703734

24. Ferrini RL, Barrett-Connor E. Sex hormones and age: a crosssectional study of testosterone and estradiol and their bioavailable fractions in community-dwelling men. Am J Epidemiol. 1998;147(8):750-4. doi: http://dx.doi.org/10.1093/oxfordjournals. aje.a009519

25. Curcio F, Ferro G, Basile C, Liguori I, Parrella P, Pirozzi F, et al. Biomarkers in sarcopenia: A multifactorial approach. Exp Gerontol. 2016;85:1-8. doi: http://dx.doi.org/10.1016/j.exger.2016.09.007

26. Cruz-Jentoft AJ, Landi F, Topinkova E, Michel JP. Understanding sarcopenia as a geriatric syndrome. Curr Opin Clin Nutr Metab Care. 2010;13(1):1-7. doi: http://dx.doi.org/10.1097/ MCO.0b013e328333clcl

27. Taekema DG, Ling CH, Blauw GJ, Meskers CG, Westendorp RG, de Craen AJ, et al. Circulating levels of IGFl are associated with muscle strength in middle-aged- and oldest-old women. Eur J Endocrinol. 2011;164(2):189-96. doi: http://dx.doi.org/10.1530/ EJE-10-0703

28. Diaz Munoz GA, Cardenas Zuluaga DM, Mesa Jimenez A. [Consistency of Mini Nutritional Assessment to Identify Sarcopenia in Older Adults in Nursing Homes in Bogota, Colombia]. Nutr Hosp. 2015;32(1):270-4. Consistencia del mini nutritional assessment para identificar la sarcopenia en adultos mayores de hogares geriatricos de Bogotá, Colombia. doi: http:// dx.doi.org/10.3305/nh.2015.32.1.8816

29. Krokstad S, Ding D, Grunseit AC, Sund ER, Holmen TL, Rangul $\mathrm{V}$, et al. Multiple lifestyle behaviours and mortality, findings from a large population-based Norwegian cohort study - The HUNT Study. BMC public health. 2017;17(1):58. doi: http://dx.doi. org/10.1186/s12889-016-3993-x

30. Farhud DD. Impact of Lifestyle on Health. Iran J Public Health. 2015;44(11):1442-4.

31. Abeytua Jiménez M, Castillo Martín JI, Martínez Castellanos T, Fernández González A, Ortega MÁ, Davalillo C, et al. Valoración del test de los seis minutos marcha y fracción de eyección del ventrículo izquierdo en pacientes que realizan programa de rehabilitación cardiaca. Rev Esp Cardiol. 2011;64 (Supl 3):375. 